\title{
Clima organizacional y su influencia en el desempeño del personal en una empresa de servicio
}

\author{
Clara Brito-Carrillo(1)* ${ }^{(12}$ emedios Pitre-Redondo(2), y Diego Cardona-Arbeláez ${ }^{(3)}$ \\ (1) Facultad de Ciencias Sociales, docente de la Universidad de la Guajira, Riohacha - Colombia \\ (correo-e: clarabrito@uniguajira.edu.co). \\ (2) Facultad de trabajo social, Universidad de la Guajira; Riohacha - Colombia. (correo-e: rpitre@uniguajira.edu.co). \\ (3) Facultad de ciencias económicas, Líder del grupo de investigación GISEMA de la Universidad Libre; Cartagena - \\ Colombia. (correo-e: diego.cardona@unilibrectg.edu.co).
}

* Autor a quien debe ser dirigida la correspondencia

Recibido Jun. 27, 2019; Aceptado Ago. 26, 2019; Versión final Oct. 2, 2019, Publicado Feb. 2020

\section{Resumen}

La presente investigación aborda el clima organizacional desde las variables liderazgo, toma de decisiones, motivación y control. El objetivo de la investigación es identificar como las variables seleccionadas influyen en el desempeño de los colaboradores en la empresa estudiada. En primera instancia se han recopilados los principales aportes de la comunidad académica en materia de clima organizacional. Luego se aplicó una encuesta basada en las variables a estudiar y posteriormente se ha realizado el análisis de correspondencia donde se determina como se relacionan las variables del estudio. Una de las principales conclusiones es que la integración de las variables control, seguimiento y liderazgo, es decisiva para la generación de un clima organizacional adecuado. Esto a su vez, influye en el desempeño y la consecución de los objetivos de las organizaciones.

\section{Organizational climate and its influence on the performance of employees in a service company}

\begin{abstract}
This research approaches the organizational climate from the variables leadership, decision-making, motivation and control. The objective of the research is to identify how the selected variables influence the performance of the collaborators in the company studied. In the first instance, the main contributions of the academic community on organizational climate have been compiled. Then, a survey based on the variables to be studied was applied and subsequently the correspondence analysis was carried out determines how the study variables are related. One of the main conclusions is that the integration of the variables control, monitoring and leadership, is decisive for the generation of an adequate organizational climate. This in turn influences the performance and achievement of the goals of organizations.
\end{abstract}




\section{INTRODUCCIÓN}

El pensamiento y la filosofía de las empresas siempre han girado en torno a la consecución de metas de orden económico, a través de diversos modelos que buscan garantizar la supervivencia y su rentabilidad, basados en diversas propuestas teóricas como es la gerencia de la calidad (Antony et al., 2005); otra es la teoría de recursos y capacidades (Barney 1991); y así mismo la teoría en torno a la gestión del conocimiento (Nonaka y Takeuchi 1995) y (Eppinger, et al., 1994). Estas propuestas promueven la manera más adecuada en que las organizaciones pueden mejorar su desempeño; sin embargo, ninguno de estos constructos teóricos puede dar los mejores resultados sin la apropiada gestión del recurso humano. En este sentido, (Tzafrir et al., 2004) afirma que las acciones orientadas al talento humano generan varias consecuencias tangibles e intangibles en las organizaciones; es por eso que la gestión del recurso humano (GRH) debe velar por la generación de canales de comunicación y transparencia en los procedimientos, lo que reduce la incertidumbre de los colaboradores y aumenta la confianza en la organización. Al respecto, Al (Mehrzi, y Singh, 2016) aseguran que es objetivo de la GRH proponer herramientas que faciliten a los colaboradores comprometerse con los objetivos y proyectos de la organización. Por su parte (Taneja et al., 2015) concluyen que la gestión de talento humano debe estar alineada con las principales estrategias organizacionales, la misión, la visión, los valores y el estilo de liderazgo, para que exista una única dirección que oriente al recurso humano. Los autores citados coinciden en indicar que el deber de la gerencia es mantener comprometidos a los empleados, por lo que es deseable que las empresas aseguren la implementación de recursos que garanticen la promoción de la cultura de compromiso organizacional tal como lo exponen (Findley et al., 2014).

En ese orden de ideas, las organizaciones entienden que un ambiente laboral positivo facilita el alcance de las metas propuestas (Sanders y Reuver, 2008), por tal motivo las empresas deben velar por garantizar el mejor clima organizacional, ya que repercute en la forma como los empleados perciben la organización y se comprometen con ella (Dimitriades, 2007). Un adecuado ambiente en las organizaciones puede conseguir una mayor vinculación del recurso humano y un mayor esfuerzo; es decir, es mantener a los colaboradores involucrados e identificados con su rol organizacional, para que desarrollen una actitud positiva hacia los cargos que desempeñan y lograr una conexión emocional positiva como exhorta (Anitha, 2014), quien también propone un modelo, que demuestra como los empelados comprometidos, se convierten en ejemplo de motivación para los otros empleados; el modelo se compone de siete factores: bienestar en el lugar de trabajo, políticas de la organización, compensación, formación y desarrollo profesional, relaciones interpersonales, liderazgo y clima o ambiente de trabajo (ver fig. 1); así mismo, las organizaciones que fomentan un adecuado clima o ambiente de trabajo, se preocupan por las necesidades, emociones de sus colaboradores y por lo tanto buscan crear espacios de retroalimentación positiva.

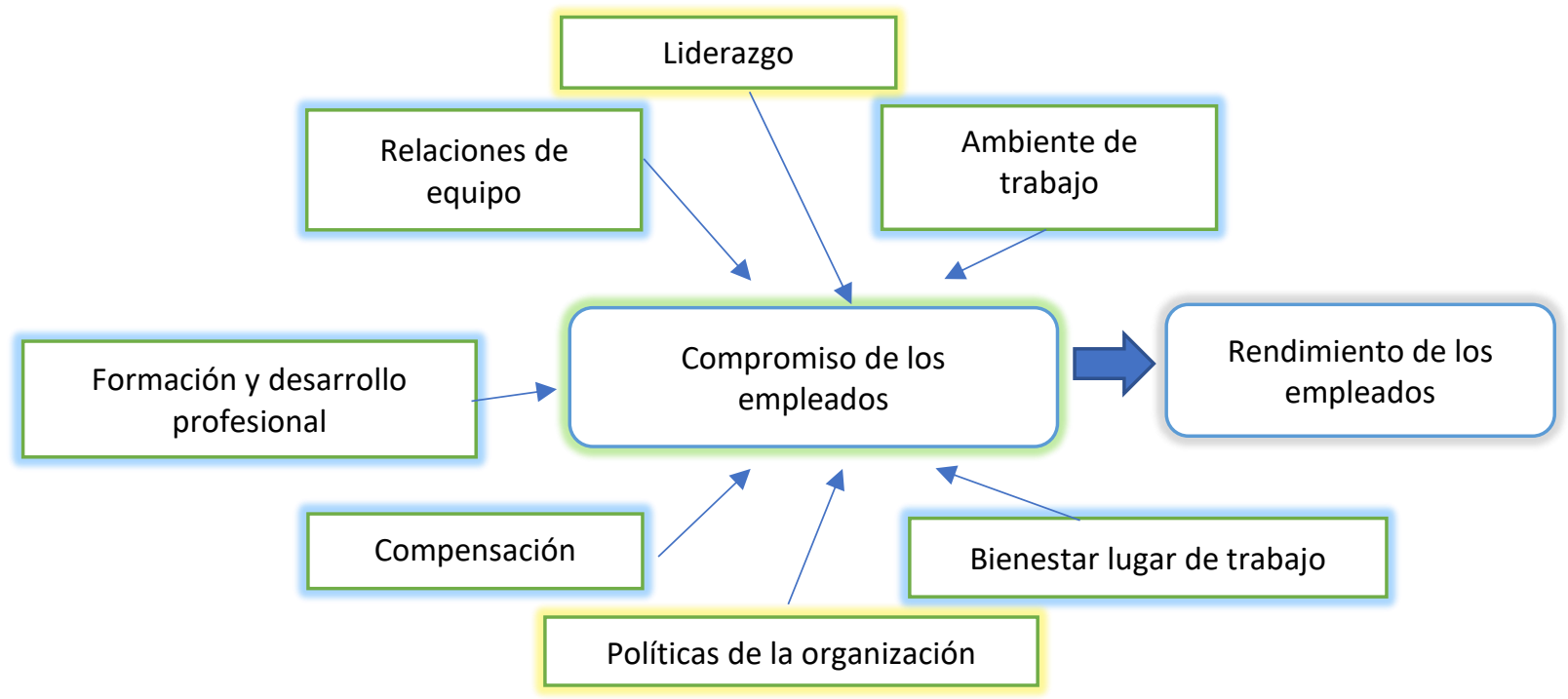

Fig. 1: Modelo desempeño del empleado a partir de su compromiso (Anitha, 2014)

Marulanda, López y Cruz (2018) coinciden con Anitha (2014), respecto a considerar que un adecuado ambiente organizacional influye en el desempeño de los colaboradores. Por tal motivo, es indispensable estudiar y analizar el clima organizacional. Lapo y Bustamante (2018) señalan que el CO se encuentra constituido por diversos factores los cuales inciden directamente en el desarrollo de comportamientos sociales, así mismo afectan los elementos mediadores de las actitudes laborales; del mismo modo Pacheco y Rodríguez (2019), consideran que el clima organizacional es concebido como la mezcla en la cual se conjugan diversos factores interrelacionados, que afectan la percepción o interpretación de los colaboradores, 
frente a las labores o roles que los mismo desempeñan en las organizaciones. Las perspectivas del concepto propuestas anteriormente permiten observar que esencia, el clima organizacional está compuesto por diversos elementos, ya sean rasgos o factores (elementos característicos o diferenciales), los cuales son percibidos subjetivamente por lo colaboradores. Dichos rasgos o factores están representados por variables, que en su totalidad y separadamente tienen conexión con la estabilidad del clima en las empresas; al respecto Méndez (2005), en su estudio "clima organizacional en Colombia: IMCOC: un método de análisis para su intervención", propone nueve variables: Identidad, Cooperación, Liderazgo, Toma de decisiones, Relaciones interpersonales, Motivación, Control, Comunicación y Proceso de Cambio.

\section{METODOLOGÍA}

La investigación es de corte descriptivo y correlacional, su objetivo es identificar como es percibido el clima organizacional (a través de las variables: liderazgo, toma de decisiones, motivación y control) por parte de los colaboradores y como dicha percepción afecta el desempeño de los mismos; en una primera fase se realiza la revisión de las fuentes secundarias relacionadas con el eje temático del estudio, seguidamente se implementa un instrumento a través del cual se mide el clima organizacional y posteriormente se realiza el análisis de correspondencia, con el fin de determinar la relación entre las variables a estudiar. Las variables seleccionadas para el desarrollo del estudio cuentan con sus respectivas unidades de análisis (Ver tabla 3 ) a través de las cuales se pudo estructurar un instrumento que se aplicó a la muestra de estudio. De otra parte, la población estudiada está constituida por 90 colaboradores de los cuales 75 son operativos y 16 administrativos, para los cuales las muestras fueron 63 y 15 respectivamente, dichas muestras fueron calculada tomando a consideración un margen de error $5 \%$ y un nivel de confianza $95 \%$ y la fórmula estadística implementada para el cálculo es la siguiente:

$n=\frac{N * Z^{2} * p * q}{e^{2} *(N-1)+Z^{2} * p * q}$

En esta ecuación, $N=$ población; $Z=$ nivel de confianza; $p=$ probabilidad de ocurrencia; $q=$ probabilidad de no ocurrencia; $e=$ margen de error. Las tablas 1 y 2 contienen los resultados obtenidos en el marco muestral estratificado, a partir de la proporción calculada de 0,83848 para administrativos y 0,96242 para los operativos (resultante de dividir la muestra general entre la población general) multiplicada por cada población de los estratos, se obtiene la muestra estratificada.

Tabla 1. Muestreo estratificado administrados

\begin{tabular}{|l|r|r|r|}
\hline \multicolumn{4}{|c|}{ Administrativos } \\
\hline Ciudad & $N$ & \multicolumn{1}{c|}{$P$} & \multicolumn{1}{c|}{$N$} \\
\hline Bogotá & 50 & 0,84 & 42 \\
\hline Medellín & 23 & 0,84 & 19 \\
\hline Cali & 2 & 0,84 & 2 \\
\hline Total & 75 & & 63 \\
\hline
\end{tabular}

Tabla 2. Muestreo estratificado administrados

\begin{tabular}{|l|r|r|r|}
\hline \multicolumn{4}{|c|}{ Operativos } \\
\hline Ciudad & $N$ & $P$ & $N$ \\
\hline Bogotá & 0 & 0,93 & 0 \\
\hline Medellín & 12 & 0,93 & 11 \\
\hline Cali & 4 & 0,93 & 4 \\
\hline Total & 16 & & 15 \\
\hline
\end{tabular}

Méndez (2005) afirma que el clima organizacional en las empresas colombianas se puede ver afectado por la cultura de las organizaciones (modo en que la empresa hace las cosas), siendo esta última resultado de la implementación de los modelos administrativos. Como ya se ha indicado Méndez propone un modelo para evaluar el clima organizacional basado en 9 variables, las cuales guardan estrecha relación con el modelo planteado por (Anitha, 2014). La Tabla 3 muestra una selección de variables, según Méndez. 
Tabla 3. Definición de variables de acuerdo con (Méndez, 2005)

\begin{tabular}{|c|c|c|}
\hline Variable & Definición & Unidad De Análisis \\
\hline Liderazgo & $\begin{array}{l}\text { Se vincula con la forma en que los superiores ejercen autoridad y la } \\
\text { forma como dicha autoridad es percibida, el efecto del liderazgo se } \\
\text { observa mediante la conducta o comportamiento de los superiores } \\
\text { hacia sus colaboradores. Esta variable se convierte en medio para } \\
\text { analizar la interacción de los jefes con sus empleados. }\end{array}$ & $\begin{array}{l}\text { Estimulo } \\
\text { Orientación }\end{array}$ \\
\hline $\begin{array}{l}\text { Toma de } \\
\text { decisiones }\end{array}$ & $\begin{array}{l}\text { Esta variable guarda relación con el modelo de dirección que } \\
\text { establece la gerencia para la consecución de las metas establecidas. } \\
\text { La toma de decisiones tiene una gran incidencia en el clima } \\
\text { organizacional, ya que permea todas las áreas en las } \\
\text { organizaciones. }\end{array}$ & $\begin{array}{l}\text { Responsabilidad } \\
\text { Capacidad }\end{array}$ \\
\hline Motivación & $\begin{array}{l}\text { Ayuda a identificar los factores que incentivan a los colaboradores y } \\
\text { los comportamientos resultantes de dicho fenómeno. } \\
\text { Existen diversos mecanismos empleados por las organizaciones } \\
\text { para motivar a los colaboradores, desde los incentivos económicos } \\
\text { hasta el reconocimiento público e inclusive a través de espacios de } \\
\text { participación en la toma de decisiones en la empresa, } \\
\text { lastimosamente en muchos casos los colaboradores de bajo niveles } \\
\text { jerárquicos no suelen tener la posibilidad de participar en la toma } \\
\text { decisiones, lo que finalmente los desmotiva (Cardona, Lamadrid \& } \\
\text { Brito, 2018). Esta variable permite conocer el nivel de satisfacción de } \\
\text { los empleados ante estímulos de motivación que la empresa genera } \\
\text { en ellos. }\end{array}$ & $\begin{array}{l}\text { Reconocimiento } \\
\text { Satisfacción }\end{array}$ \\
\hline Control & $\begin{array}{l}\text { Busca mantener a los colaboradores alineados para el cumplimiento } \\
\text { de los indicadores productivos, además que funciona como método } \\
\text { para la corrección de anomalías en el desempeño. } \\
\text { El manejo inadecuado de esta variable incide negativamente en la } \\
\text { percepción de los colaboradores en el ámbito laboral. }\end{array}$ & $\begin{array}{l}\text { Eficiencia } \\
\text { Acompañamiento de } \\
\text { los superiores }\end{array}$ \\
\hline
\end{tabular}

\section{ANÁLISIS DE CORRESPONDENCIA}

El análisis de correspondencia múltiple (ACM), se introduce como técnica gráfica, que minimiza las distancias entre los puntos que representan la relación de las variables dentro de un modelo Hoffman y Leeuw (1992). Al respecto, Fernández (2011) explica, el análisis de correspondencia múltiple es una técnica descriptiva y exploratoria, cuya meta es reducir las grandes cantidades de datos en un número más manejable o en dimensiones, se refiere al agrupamiento de variables con la menor perdida de información posible. El análisis de correspondencia múltiple es similar al método de factoriales, salvo que ACM se aplica a variables de categoría o de orden. Como parte de los resultados de la implementación del ACM, se obtuvo distintas figuras y tablas, dentro de las cuales se encuentra el resumen del modelo, el cual cuenta con los coeficientes de fiabilidad para las dos dimensiones empleadas en el estudio, además del coeficiente de inercia. (ver tabla. 4)

Tabla 4. Resumen del modelo

\begin{tabular}{|c|c|c|c|c|}
\hline \multirow{2}{*}{ Dimensión } & \multirow{2}{*}{ Alfa de Cronbach } & \multicolumn{3}{|c|}{ Varianza contabilizada para } \\
\cline { 3 - 5 } & & Total (autovalor) & Inercia & $\%$ de varianza \\
\hline 1 & 0,922 & 5,171 & 0,646 & 64,642 \\
\hline 2 & 0,568 & 1,986 & 0,248 & 24,83 \\
\hline Total & & 7,158 & 0,895 & \\
\hline Media &, $824^{\text {a }}$ & 3,579 & 0,447 & 44,736 \\
\hline
\end{tabular}

Ahora bien, la gráfica 2 muestra cómo se distribuyen las dimensiones en el ACM, además de la relación existente entre ellas, en la medida en que una variable se encuentre cercana a otra se puede asegurar que son variables con un alto coeficiente de relación. Del mismo modo la gráfica permite ver que existen dos grupos de unidades de análisis relacionadas entre sí, el primer grupo se encuentra conformado por las variables liderazgo (unidad de análisis, estimulo), de la cual se obtuvo los coeficientes 0,537 para la dimensión 1 y 0,452 para la dimensión 2; y toma de decisiones (unidad de análisis, capacidad), muestra los coeficientes 0,537 para la dimensión 1 y 0,452 para la dimensión 2. EL segundo grupo de variables se encuentran relacionado con el liderazgo (unidad de análisis, orientación), cuyos coeficientes resultantes son 0,787 para la dimensión 1 y 0,098 para la dimensión 2; la variable motivación (unidad de análisis reconocimiento) muestra coeficientes de 0,507 para la dimensión 1 y 0,451 para la dimensión 2; respecto a la variable control en tanto a la unidad de análisis (eficiencia), se obtuvieron los coeficientes 0,541 para la dimensión 1 y 0,162 para la dimensión 2. 


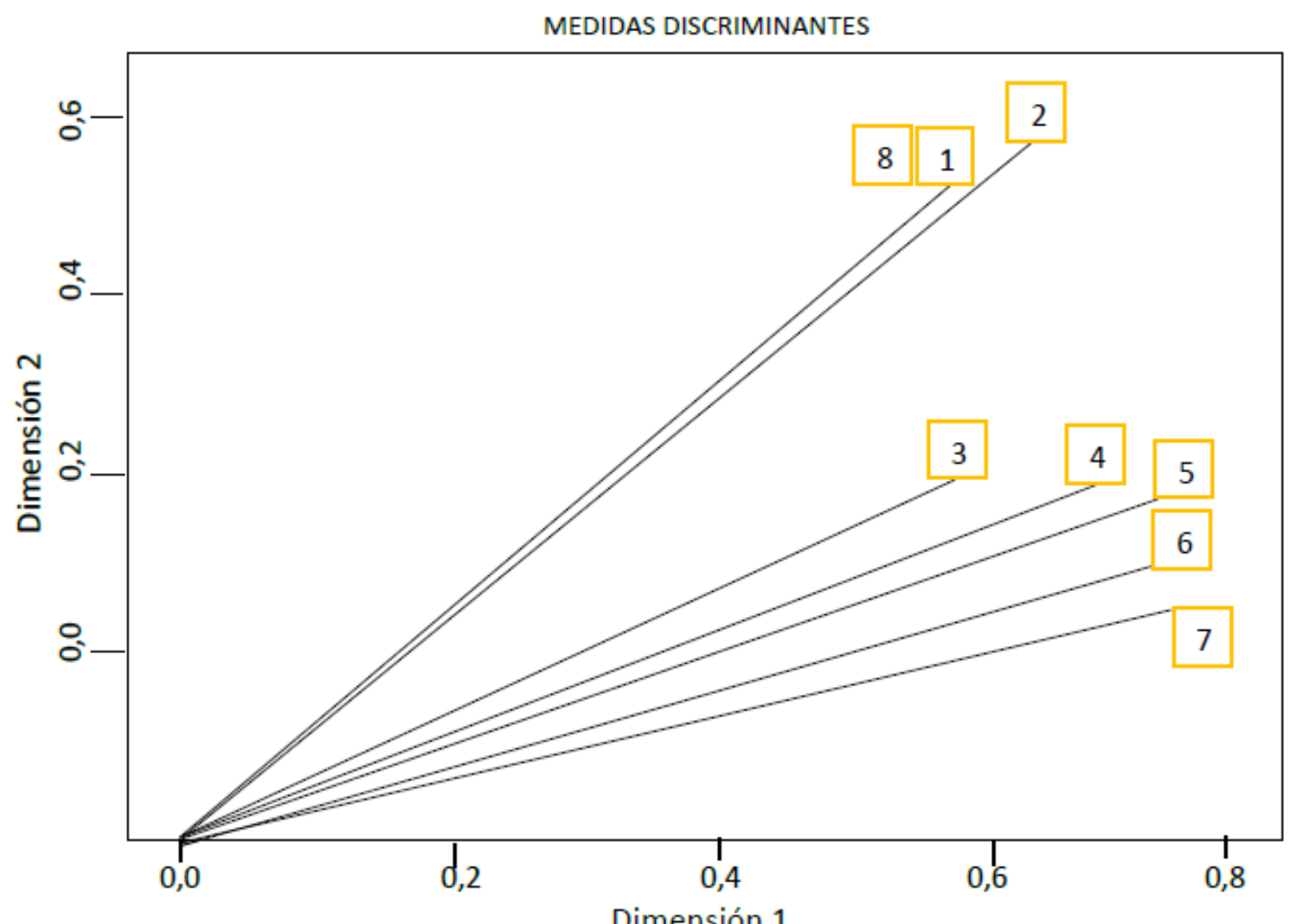

1. Siento que puedo hacerme cargo de nuevas

responsabilidades en mi trabajo además de las que tengo

5. Recibo retroalimentación de los resultados de mi trabajo como forma de evaluación.
2. Siento que tengo autonomía para realizar mi trabajo.

6. Conozco las metas y objetivos de trabajo establecidas periódicamente por mi jefe
3. Estoy de acuerdo con 4 la forma e indicadores con que se evalúa mi trabajo.

7. Estoy satisfecho porque el salario que percibo es justo frente al trabajo que realizo
4. Creo que mi jefe inmediato me tiene en cuenta al tomar una decisión que me afecte.

8. La empresa distingue $y$ premia públicamente a los trabajadores que tienen buen desempeño en su trabajo.

Fig. 2: medidas discriminantes

La tabla 4 muestra detalladamente los coeficientes obtenidos para cada afirmación, lo que permite ubicar cada variable vinculada en una de las dos dimensiones del análisis de correspondencia múltiple. La razón para indicar que existen dos grupos de unidades de análisis se sustenta en el vínculo de las misma a través los coeficientes de sus medidas discriminantes, como se puede observar es un grupo pequeño el que tienen la tendencia más equilibrada mostrando coeficientes cercanos para ambas dimensiones, el segundo grupo es más grande está compuesto por unidades de análisis de las cuatro variables y muestran una mayor tendencia a la dimensión 1, lo anteriormente explicado se puede constatar en la tabla 5.

Tabla 5. Tabla detallada medidas discriminantes

\begin{tabular}{|c|c|c|c|}
\hline & \multicolumn{2}{|c|}{ Dimensión } & \multirow{2}{*}{ Media } \\
\hline & 1 & 2 & \\
\hline Siento que tengo autonomía para realizar mi trabajo & ,537 & ,452 & ,494 \\
\hline Conozco las metas y objetivos de trabajo establecidas periódicamente por mi jefe & ,787 & 098 & ,442 \\
\hline $\begin{array}{l}\text { Siento que puedo hacerme cargo de nuevas responsabilidades en mi trabajo además de las } \\
\text { que tengo actualmente }\end{array}$ & ,537 & ,452 & 494 \\
\hline Creo que mi jefe inmediato me tiene en cuenta al tomar una decisión que me afecte & ,733 & , 169 & 451 \\
\hline $\begin{array}{l}\text { La empresa distingue y premia públicamente a los trabajadores que tienen buen } \\
\text { desempeño en su trabajo }\end{array}$ &, 507 & ,451 & 479 \\
\hline Estoy satisfecho porque el salario que percibo es justo frente al trabajo que realizo & ,757 & 051 & ,404 \\
\hline Estoy de acuerdo con la forma e indicadores con que se evalúa mi trabajo & ,541 & ,162 & ,351 \\
\hline Recibo retroalimentación de los resultados de mi trabajo como forma de evaluación & ,773 & 152 & 462 \\
\hline Total activo & 5,171 & 1,986 & 3,579 \\
\hline
\end{tabular}




\section{RESULTADOS Y DISCUSIÓN}

De acuerdo con los hallazgos del estudio, se puede observar que las dimensiones estimulo y orientación son percibidas por los colaboradores como favorables para el clima de la organización, ello queda evidenciado, en los resultados obtenidos, ya que ambas unidades de análisis poseen porcentajes de respuesta por encima del $50 \%$ y $25 \%$, para las opciones de respuesta de acuerdo y muy de acuerdo respectivamente. De igual modo se obtiene porcentajes iguales o superiores al 18\% en la opción de respuesta Indeciso, lo cual denota que para una pequeña parte de los encuestados les es difícil emitir una evaluación referente al estilo de liderazgo de la organización; de igual modo se observan porcentajes de $0 \%$ para las opciones en desacuerdo y muy en desacuerdo (ver fig. 3).

En lo referente a los resultados obtenidos para la variable, toma de decisión muestran que las dimensiones Responsabilidad y capacidad son evaluadas con porcentajes iguales o por encima del $70 \%$ en las opciones de repuesta de acuerdo lo que evidencia que la gerencia proyecta imagen de ser responsable y de poseer las capacidades necesarias para tomar las mejores decisiones teniendo en cuenta el bienestar de sus colaboradores; tan solo se obtiene un $18 \%$ y $26 \%$ para la opciones de respuesta indeciso para las dimensiones que componen la variable (ver fig. 4). En términos generales se observa que el estilo de liderazgo de los directivos ayuda a la construcción de un ambiente adecuado, en el cual los empleados pueden desarrollar con mayor autonomía los roles que les han sido asignados. Lo anterior va de la mano del compromiso que proyecta la empresa por ser más responsables con las acciones que desarrolla y que afectan el desempeño de todos los empleados. Es correcto indicar que ambas variables son fortalezas de la empresa.

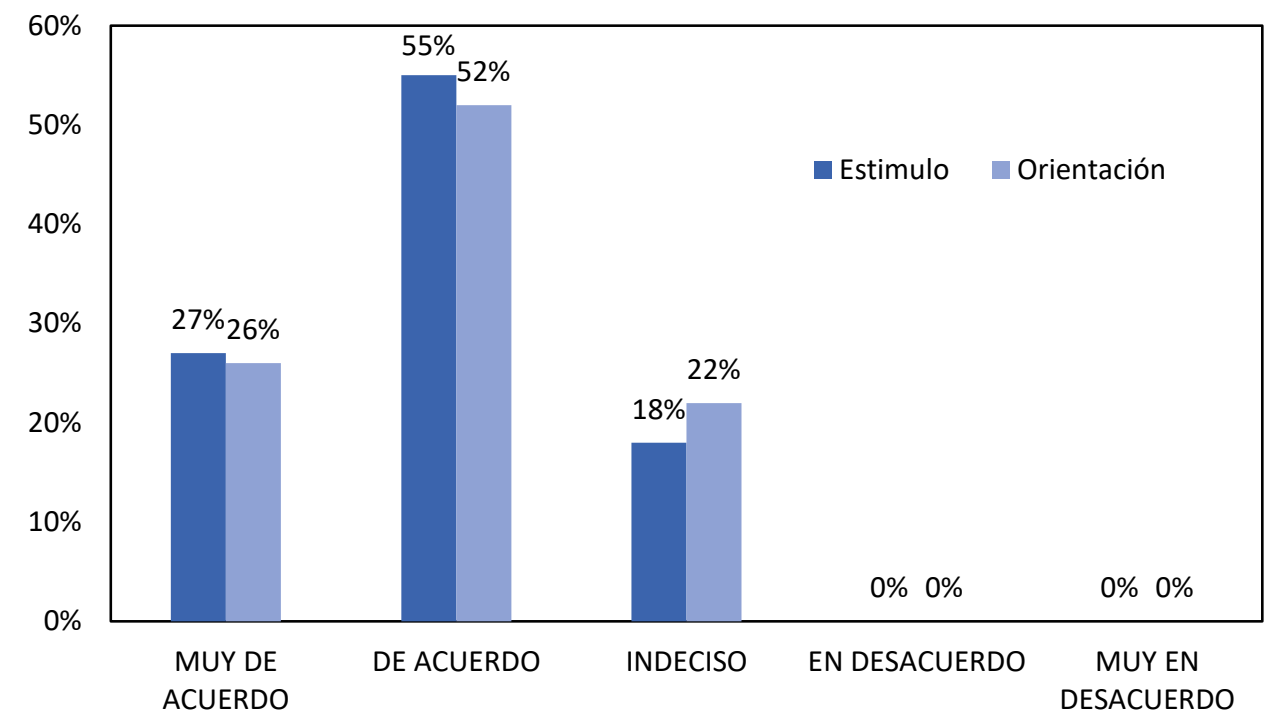

Fig. 3: Variable Liderazgo

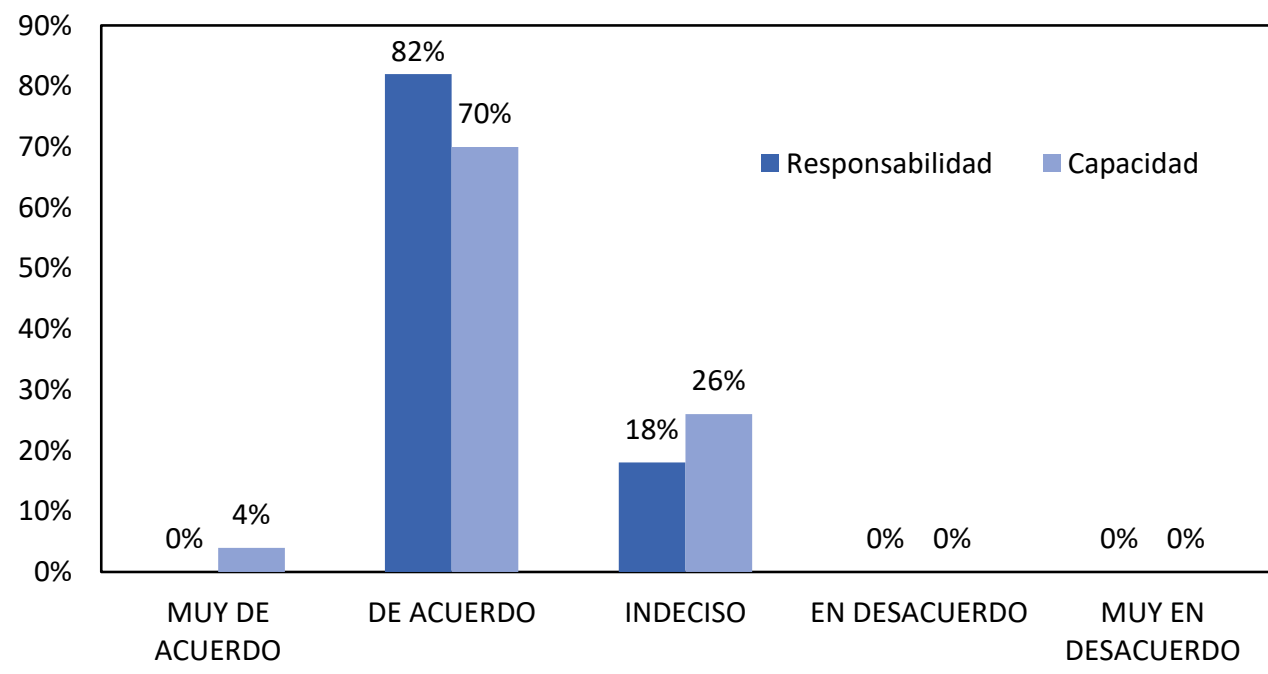

Fig. 4: Variable Toma de decisiones 
Los resultados obtenidos para la variable motivación permite ver una respuesta positiva por parte de los encuestados, ya que se obtiene porcentaje de respuesta por encima del 49\% (tanto para la dimensión reconocimiento como para satisfacción) lo que denota que la gerencia motiva a sus colaboradores, sin embargo, cabe indicar que un pequeño porcentaje evalúa negativamente la variable con una representación de más de 12 puntos porcentuales, tanto para las unidades de análisis reconocimiento como para satisfacción, una posible razón para este resultado puede estar vinculada a una inadecuada percepción de la variable control. (ver fig. 5). Los resultados de la variable control, muestran que existe una mayor tendencia al desarrollo de mecanismos para medir los resultados, dejando de lado el acompañamiento de la gerencia hacía sus colaboradores, factor relevante para la consecución de las metas; lo anterior se observa a través $29 \%$ de los encuestados evalúan negativamente la dimensión de acompañamiento por parte de sus superiores. (ver fig. 6)

Respecto a las otras variables estudiada en la investigación, la variable control es la que demanda mayor atención por parte de la gerencia, pues esta incide en los estados anímicos mostrados por los empleados y en consecuencia en las bajas del desempeño e inclusive en el compromiso de los mismo para con los objetivos y metas propuestas por la gerencia. Lo más recomendable es que se reformule el modelo de seguimiento y acompañamiento, con la finalidad que los colaboradores se sienta más apoyados por parte de la gerencia. La falta de elementos que faciliten la interacción, integración y guía de la gerencia a los colaboradores, constituyen un factor fundamental que desmotiva al personal; la gerencia debe implementar procesos de seguimiento, guía, acompañamiento y control, bajo el concepto de integralidad.

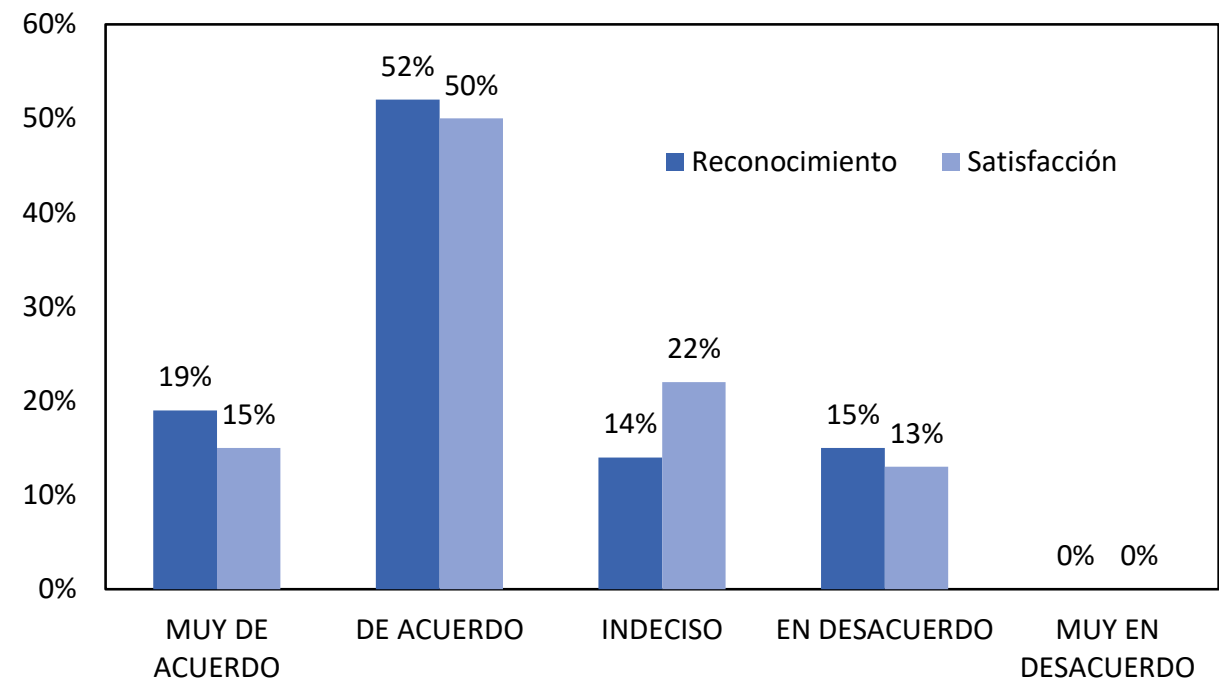

Fig. 5: Variable Motivación

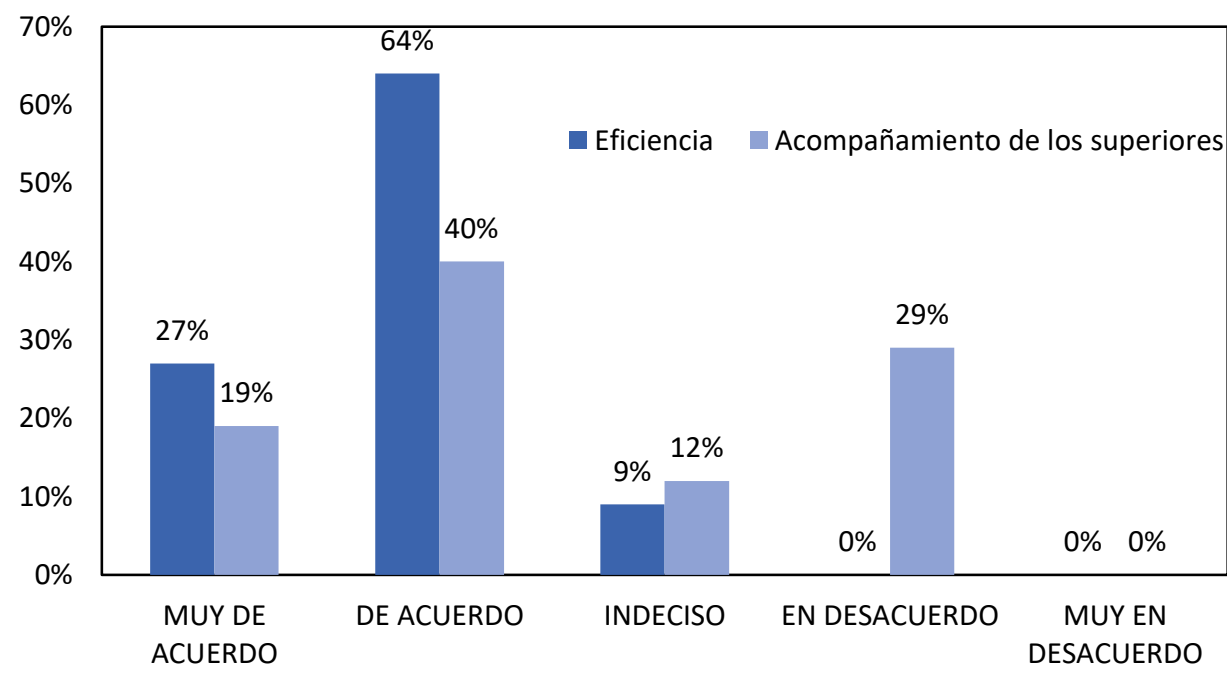

Fig. 6: Variable Control 


\section{CONCLUSIONES}

Partiendo de los resultados obtenidos en la investigación, se puede concluir: 1) Los planes y/o programas de acompañamiento a los colaboradores, deben implementar canales de comunicación efectivos, a través de cuales se manejen tanto las inquietudes como las sugerencias producto de la actividad productiva diaria. 2) La integración de las variables control, seguimiento y liderazgo, es decisiva para la generación de un clima organizacional adecuado, lo que a su vez incide en el desempeño y la consecución de los objetivos de las organizaciones. 3) Propiciar espacios de participación en la toma de decisiones es un factor que influye en la motivación y en el desempeño de los colaboradores. 4) Cultivar en los colaboradores confianza aumenta empoderamiento y compromiso de los mismo para afrontar los retos presentes y futuros. 5) La gerencia debe garantizar los recursos necesarios para construir y mantener el clima organizacional más adecuado, que facilite el óptimo desempeño de los colaboradores.

\section{REFERENCIAS}

Al Mehrzi, N., S. K. Singh, Competing through employee engagement: a proposed framework, doi: https://doi.org/10.1108/IJPPM-02-2016-0037, International Journal of Productivity and Performance Management, 65(6), 831. 843 (2016)

Anitha, J., Determinants of employee engagement and their impact on employee performance, doi: 10.1108/IJPPM-01-20130008, International journal of productivity and performance management, 63(3), 308 (2014)

Antony, J., M. Kumar, y C. N. Madu, Six sigma in small-and medium-sized UK manufacturing enterprises: Some empirical observations, doi: 10.1108/02656710510617265, International Journal of Quality \& Reliability Management, 22(8), 860-874 (2005)

Barney, J., Firm resources and sustained competitive advantage. Journal of management, doi: 10.1177/014920639101700108, 17(1), 99-120 (1991)

Cardona, D. A., J. L. Villarreal, y C. J. B. Carrillo, La gestión y dirección del talento humano desde el análisis sobre clima organizacional y sus dimensiones. Un estudio de caso, doi: 10.22519/22157360.1185, Aglala, 9(1), 154-176 (2018)

Dimitriades, Z. S., The influence of service climate and job involvement on customer-oriented organizational citizenship behavior in Greek service organizations: a survey, doi: 10.1108/01425450710776290, Employee Relations, 29(5), 469-491 (2007)

Eppinger, S. D., D. E. Whitney, R. P. Smith, y otro autor, A model-based method for organizing tasks in product development, doi: 10.1007/BF01588087, Research in engineering design, 6(1), 1-13 (1994)

Fernández, R. S. M., Análisis de correspondencias simples y múltiples. Universidad Autónoma de Madrid: Facultad de Ciencias Económicas y Empresariales (2011)

Findley-Musgrove, C., E. Ellinger, y D. Ellinger, Examining the influence of strategic profit emphases on employee engagement and service climate, doi: 10.1108/JWL-08-2013-0057, Journal of Workplace Learning, 26(3/4), 152-171 (2014)

Hoffman, D. L., J. De Leeuw, Interpreting multiple correspondence analysis as a multidimensional scaling method, doi: 10.1007/BF00994134, Marketing letters, 3(3), 259-272 (1992)

Lapo-Maza, M. D. C., M. A. Bustamante-Ubilla, Incidencia del Clima Organizacional y de las Actitudes Laborales en el Comportamiento Prosocial de los Profesionales de la Salud del Guayas Ecuador, doi: 10.4067/S0718-07642018000500245, Información tecnológica, 29(5), 245-258 (2018)

Marulanda, C., L. López., y G. Cruz, La Cultura Organizacional, Factor Clave para la Transferencia de Conocimiento en los Centros de Investigación del Triángulo del Café de Colombia, doi: 10.4067/S0718-07642018000600245, Información tecnológica, 29(6), 245-252 (2018)

Méndez-Álvarez, C., Clima organizacional en empresas colombianas 1980-2004, Universidad \& Empresa, 7(9), 100-121 (2010)

Nonaka, I., H. Takeuchi, The knowledge-creating company: How Japanese companies create the dynamics of innovation. Oxford university, United Kingdom (1995)

Pacheco, S. L., D. A. C. Rodríguez, Propuesta pedagógica para el mejoramiento del clima organizacional, doi: 10.15665/encuent.v17i01.1674, Encuentros, 17(1), 145-161 (2019)

Sanders, K., L., Dorenbosch, y R. de Reuver, The impact of individual and shared employee perceptions of HRM on affective commitment: Considering climate strength, doi: 10.1108/00483480810877589, Personnel Review, 37(4), 412-425 (2008)

Taneja, S., S. S. Sewell, y R. Y. Odom, A culture of employee engagement: A strategic perspective for global managers, doi: 10.1108/JBS-06-2014-0062, Journal of Business Strategy, 36(3), 46-56 (2015)

Tzafrir, S. S., H. late-Gedaliahu, Y. Harel, Baruch, y otro autor, The consequences of emerging HRM practices for employees' trust in their managers, doi: 10.1108/00483480410561529, Personnel Review, 33(6), 628-647 (2004) 